\title{
EFFETS RESPIRATOIRES ET HEMODYNAMIQUES DE L'ANESTHESIE AU METHOXYFLURANE
}

\author{
F. HUdON, F.R.C P.(C), F.F.A.R.C.S., A. JACQUES, F.R.C.P.(C), R. DÉRY, F.R C.P.(c), \\ J. RouX, M D., ET J. MÉNard, M D.
}

Après trois ans d'un emploi de plus en plus répandu en anesthésie clinique, le méthoxyflurane demeure un produit digne du plus grand intérêt. Certains avantages indiscutables ont contríbué à cette renommée: stabilité, puissance hypnotique et analgésique, faible toxicité, chez un agent anesthésique non explosif et doué de propriétés myo-résolutives comparables à celles de l'éther di-éthylique. Cependant, même sı la plupart des anesthésistes sont maintenant familiers avec la pharmacologie et le mode d'administration de ce produit, d'autres se refusent encore à l'utiliser parce qu'il s'agit d'une substance trop nouvelle et controversée. Leur adhésion ne sera obtenue que lorsque le méthoxyflurane, après avoir été soumis à une épreuve concluante par les chercheurs expérimentaux et cliniques, aura livré tous ses secrets.

Certains auteurs ont rapporté que le méthoxyflurane provoque de l'hypotension, une baisse du débit cardiaque et une diminution de la résistance périphérique., $1,2,318$ Comme notre impression clinique ne confirmait pas ces données expérimentales, généralement obtenues chez des chiens ou chez un nombre nettement insuffisant de patients, nous avons entreprus d'élucider les effets hémodynamiques de l'anesthésie au méthoxyflurane. Au cours de ces recherche's faites dans la salle d'opération, nous avons été amenés à étudier l'action du produit sur la ventilation et sur l'équilibre acide-base. Nous inclurons ces données additionnelles dans nos résultats.

\section{MÉTHODE DE TRAVALL}

Notre étude a porté sur deux groupes de patients. Le premier groupe, que nous avons appellé "groupe respiratoire," comprend 18 patients d'âge adulte qui ont été laissés en respiration spontanée tout au long de leur anesthésie au méthoxyflurane. Chez ces patients, en plus de mesurer la ventilation pulmonaire avec le ventimètre de Wright, nous avons inséré avant l'anesthésie un trocart dans une artère humérale, ce qui nous a permis de suivre les répercussions de l'administration du méthoxyflurane sur la $p \mathrm{O}_{2}$, la $p \mathrm{CO}_{2}$ et le $\mathrm{pH}$ artériel. Le $\mathrm{CO}_{2}$ total a été déterminé d'après le nomogramme de McLean, ${ }^{5}$ tandis que le diagramme de Davenport ${ }^{b}$ nous a servi à apprécier l'accumulation possible d'acides fixes dans le sang.

Notre deuxième groupe de patients, appellé "groupe hémodynamique," com-

* Du département d'anesthésie de l'Hôtel-Dieu de Québec. Traval présenté au Congrès national de la Société Canadienne d'Anesthésie, Mai, 1963 (à Montebello, P.Q.).

Pour les figures, les tableaux et la Bıbliographie vorr l'artıcle précédent. 
prend pour sa part 23 patients en excellente condition physique. Trois cependant (patients $5,6,7$ ) souffraient d'hypertension artérielle sans répercussion cardiaque ou rénale. Chez tous ces patients, en plus d'insérer avant l'anesthésie un trocart artériel, nous avons ponctionné une veine basilique au pli du coude avec une aiguille 15 puis nous avons inséré dans la lumière de cette aiguille un cathéter de polyéthylène que nous avons poussé jusqu’à la veine cave supérieure ou l'oreillette droite. Nous avons apprécié la localisation du bout distal du cathéter d'après la longueur insérée. Chez quelques patients par contre, à cause de difficultés techniques, nous avons prélevé les échantillons de sang veineux dans la jugulaire externe au moyen d'une aiguille ou d'un court cathéter. Ceci entraine une légère erreur dans la détermination du débit cardiaque, mais les résultats demeurent comparables entre eux chez un même patient et proportionnels à ceux obtenus d'après la méthode ci-dessus mentionnée.

La fréquence du pouls et son rythme ont été appréciés avec la Scopette de la compagnie Corbin Farnsworth, le même appareil nous permettant la surveillance de l'électroencéphalogramme. La pression artérielle a été déterminée suivant la méthode auscultatoire de Riva Rỏcci, la pression diastolique étần toujours jugée par le même observateur telle qu'indiquée par la disparition ou l'assourdissement marqué des bruits transmis. Pour déterminer le débit carrdiaque, nous ne nous sommes pas servi des courbes de dilution de substances colorantes; nous avons prêféré en rester au principe de Fick qui est encore le point de référence des autres techniques de recherche du débit cardiaqueĩ; c'est d'ailleurs la méthode qui comporte la plus basse marge d'erreur. Cependant, au lieu d'obtenir le débit cardiaque à partir de la consommation d'oxygène par minute et de la différence artérioveineuse en oxygène, nous avons préféré utiliser le débit de $\mathrm{CO}_{2}$ par minute et la différence artério-veineuse en $\mathrm{CO}_{2}$. Nous avons obtenu facilement cette différence artério-veineuse en $\mathrm{CO}_{2}$ par l'analyse des échantillons sanguins prélevés, au moyen de l'analyseur des paramètres sanguins de la Compagnie Epsco Medical (fig. 4).

Restait à déterminer le débit de $\mathrm{CO}_{2}$ par minute. Nous y sommes parvenus de la façon suivante: au moyen du capnographe de Godart (fig. 1) et de l'appareil enrégistreur qui y est annexé, nous avons obtenu la courbe traduisant la concentration de $\mathrm{CO}_{2}$ dans l'air expiré. Voici l'allure générale de cette courbe (fig. 5). En calculant au moyen d'un planimètre la surface de cette courbe et en la comparant à une surface étalon, nous avons obtenu la concentration moyenne de $\mathrm{CO}_{2}$ dans l'air expiré. Si l'on multiplie la ventilation-minute obtenue avec le ventimètre de Wright par ce pourcentage moyen de $\mathrm{CO}_{2}$, on obtient le débitminute de $\mathrm{CO}_{2}$ avec un pourcentage très bas d'erreur (des contrôles ont démontré que cette erreur se chiffrait en bas de $7 \%$ ). La figure 2 illustre les raccords nécessaires sur le circuit d'anesthésie, et la figure 3 montre la disposition de tout l'appareillage.

Pour calculer la résistance périphérique, nous nous sommes servis de la formule classique d'Aperia ${ }^{8}$ :

$\frac{\text { pression artérielle moyenne } \times 1332}{\text { débit cardiaque en cc./seconde }}=$ dynes par seconde par centimètre ${ }^{-5}$ 
Les échantillons de sang artériel et de sang veineux mêlé ont été prélevés de façon anaérobie sous huile minérale neutre et en présence d'un mélange de fluorure et d'oxalate de sodium comme anticoagulant. Après la ponction initiale effectuée avant l'anesthésie, nous avons attendụ une heure avant les prélèvements ultérieurs pour permettre au patient de se stabiliser au niveau d'anesthésie désiré et d'échapper aux effets dépresseurs résiduels du Pentothal ou aux effets stimulants de l'intubation endotrachéale. Par la suite, les prélèvements artériels et veineux ont été faits aux 30 minutes environ jusqu'au réveil complet du malade.

L'induction de l'anesthésie a été réalisée avec un mélange de 2 litres d'oxygène, 2 litres de protoxyde d'azote et des concentrations de méthoxyflurane allant jusqu'à 3 pour cent, telles qu'indiquées au vaporisateur Vernitrol ou Pentec. Mentionnons cependant que 13 patients refusant l'induction au masque ont reçu une dose de sommeil de Pentothal, après quoi le maintien de l'anesthésie a été obtenu avec des concentrations de méthoxyllurane variant entre 0.8 et 0.45 pour cent. Après quelques instants de respiration contrôlée, nos patients soumis à des interventions orthopédiques ou reconstructives ont été laissés en respiration spontanée. D'autre part, les patients qui ont subi des interventions abdominales ont reca l'assistance respiratoire nécessitée par l'administration de curares grâce à l'emploi d'un respirateur Bird à pression positive intermittente. L'air courant produit par l'appareil a généralement été identique à celui que possédait le patient ayant l'intervention, sauf dans quelquess cas où la ventilation-minute a dû être corrigée pour éviter l'hypercapnie ou l'hyperventilation. Nos patients ont présenté une anesthésie stable, dénuée de réactions à la douleur ou de réflexes nociceptifs. Mentionnons enfin que la perte sanguine estimée par la méthode gravimétrique a été couverte par du soluté mixte ou par du sang lorsque nécessaire, de façon à prévenir toute vasoconstriction d'origine hypovolémique.

\section{RÉSULTATS}

\section{Effets respiratoires}

Nous avons groupé nos résultats dans le tableau I Un seul de nos patients était en dépression respiratoire avant l'induction de l'anesthésie. Tous les autres présentaient une ventilation pulmonaire adéquate, voire même une légère hyperventilation. Un fait capital noté dans notre étude est l'absence relative de dépression respiratoire pendant l'anesthésie et au réveil chez la plupart de nos patients laissés en respiration spontanée durant l'anesthésie au méthoxyllurane. Nous avons remarqué une respiration ample et suffisante, et les déterminations en série de la tension partielle du $\mathrm{CO}_{2}$ artériel ont confirmé cette observation. Mentionnons cependant que nous avons maintenu chez ces patients un niveau d'anesthésie plutôt superficiel, et généralement situé au milieu du deuxième plan du stage chirurgical. Lorsqu'on atteint des niveaux plus profonds de narcose, l'amplitude des mouvements thoraciques diminue, le rythme se ralentit et l'acidose respiratoire apparaitt. Trois de nos patients (cas 11, 15, 18) ont présenté cette bradypnée superficielle et ont nécessité par la suite une assistance respiratoire. Aucun de nos patients par contre n'a présenté d'hypoxémlie. $\mathrm{La} \quad \mathrm{pO}_{2}$ artérielle per-opératoire s'est toujours maintenue entre 200 et $300 \mathrm{~mm}$. $\mathrm{Hg}$, ce qui est normal dès l'instant 
où l'on assưre l'apport d'un mélange contenant 50 pour cent d'oxygène. Au réveil également, nous n'avons accusé aucune baisse de la saturation oxyhémoglobinique due à l'hypoventilation, et les $p \mathrm{O}_{2}$ de nos patients jouent entre 100 et $200 \mathrm{~mm}$. $\mathrm{Hg}$ suivant que nous leur administrons ou non de l'oxygène au moment du prélèvement.

Nos études sur l'équilibre acıde-base pendant l'anesthésie au méthoxyflurane puisent également leurs données dans le tableau I. Nos résultats confirment la tendance génởrale des auteurs sur le même sujet, notamment celles de Dobkin et de ses collaborateurs. ${ }^{9} \mathrm{La}$ très légère acidose métabolique rencontrée chez certains de nos malades ne semble pas digne d'être remarquée. Le méthoxyflurane ne modifie pas à toute fin pratique la concentration des acides fixés du'sang, et la figure 6 montre que chez l'ensemble des' 18 patients étudiés sous cet aspect, la réserve de bases-tampons accuse peu de modifications.

\section{Effets cardio-vasculaires}

1. Le pouls. Une fois que le patient a atteint un niveau stable d'anesthésie et ques les effets stimulants de l'intubation ont disparu, la fréquence du -pouls demeure à peu près identiquẻ à celle notée avant l'opération. On note chez certains patients une légère accélération, de l'ordre de 8 à 10 contractions par minutes; cette accélération du pouls nous semble un effet général de l'anesthésie avec les éthers. L'effet chronotropique négatif observé par| certains auteurs au cours de l'anesthésie au méthoxyflurane nous apparaît une rareté.

2. La pression artérielle. 'De son côté, la pression artérielle a montré une grande stabilité pendant l'anesthésie, et les légères déviations notées en plus ou en moins nous semblent non significatives. La pression artérielle moyenne chez nos 23 patients se chiffrait à $103 \mathrm{~mm}$. Hg avant l'anesthésie, Ce chiffre se maintient à $101 \mathrm{~mm}$. Hg au cours de l'anesthésie. Nous en concluons qu'à des niveaux superficiels d'anesthésie, le méthoxyflurane ne cause pas de chûtes de pression, l'hypotension étant le fait d'une surdose. ${ }^{10} \mathrm{~A}$ la salle de réveil, la prëssion artérielle s'est maintenue aux niveaux antërieưrs chez la plupart de nos patients. Cependant, si nous calculons la pression artérielle moyenne de nos patients à ce moment, nous la trouvons abaissée à $92.8 \mathrm{~mm}$. Hg. Nous attribuons cette baisse surtout à trois de nos patients (cas $9,11,21$ ) qui ont souffert d'une surdose de méthoxyflurane au cours de leur anesthésie et qui ont présenté au réveil une dépression circulatoire considérable et difficilement contrôlée par l'injection de vasopresseurs légers (Mephentermine). Notons que la pression artérielle de nos hypertendus ( cas $5,6,7$ ) a connu une baisse modérée lors de l'induction au méthoxyllurane, sans doute à la suite de la vasodilatation systémique prësente à ce moment.

3. Le débit cardiaque. Les chiffres de débit cardiaque inclus dans le tableau II méritent qu'on les étudie attentivement. Dans la période pré-opératoire, le débit cardiaque chez la majorité des patients étudiés se trouve entre $4 \grave{a ̆} 6^{\natural}$ litres par minute. Nous avons exclu de ces statistiques trois patients qui ont développé de l'hypercapnie en cours d'anesthésie et dont le cas sera discuté à part. A la fin de la première heure d'anesthésie, le débit cardiaque est diminué de 19 pour cent en moyenne chez 13 de nos 20 sujets, alors que les sept autres montrent une augmentation de débit de 23 pour cent. A la fin de la deuxième heure d'anesthésie, il reste 
encore 19 patients dans la salle d'opération. De ce nombre, 15 présentent une diminution moyenne de 22 pour cent de leur débit cardiaque; les 4 autres continuent d'accuser une augmentation moyenne de 23 pour cent. A la fin de la troisième heure d'anesthésie, 13 patients sont encore sous anesthésie stable. De ces 13,10 accusent une baisse moyenne de 31.5 pour cent de leur débit cardiaque alors que seulement 3 patients continuent à maintenir leur débit cardiaque un peu en haut de leur chiffre initial. A la salle de réveil, chez les 23 patients étudiés, 10 patients montrent un débit cardiaque augmenté d'en moyenne 28 pour cent. Incidemment, on observe que ce sont précisément ces 10 patients qui présentent un réveil rapide, survenant même la plupart du temps dans la salle d'opération. Leur apparence clinique est parfaite; ils répondent tôt aux questions posées et leur peau est sèche et rosée. Treize patients par contre présentent une chûte moyenne de 37 pour cent de leur débit cardiaque au réveil, et chez 8 de ces patients, la chûte est plus importante encore. C'est précisément chez ces 8 patients que nous avons noté le tableau clinique bien connnu mais encore inexpliqué fait de pâleur, cyanose distale et réveil tardif.

4. La résistance périphérique. Les chiffres obtenus par le calcul d'Aperia sont également compris dans le tableau II. Au premier coup d'oeil, nous voyons que ces résultats sont passablement différents de ceux rapportés par d'autres auteurs. ${ }^{2,3}$ Nous n'avons pour notre part |remarqué une diminution importante de la résistance périphérique que chez 7 de nos patients, et encore cette vasodilatation s'est-elle produite à un moment bien précis, c'est-à-dire pendànt la première heure d'anesthésie, alors que le débit cardiaque était plutôt élevé. A partir de la deuxième heure et par la suite, la majorité de nos patients ont montré une augmentation de leur résistance périphérique et nous voulons souligner que cette augmentation a toujours été reliée à une baisse concommittante du débit cardiaque. Plus le débit cardiaque est bas, plus la résistance périphérique s'élève. Nous avons été fortement impressionnés par cette relation inversement proportionnelle entre le débit cardiaque et la résistance périphérique sous anesthésie au méthoxyflurane, et nous insisterons sur ce phénomène dans la discussion.

\section{Discussion}

Même si tous les auteurs s'accordent à dire que le méthoxyflurane est un puissant dépresseur de la respiration, notre expérience et nos résultats nous permettent d'affirmer qu'à des niveaux superficiels d'anesthésie chirurgicale, le méthoxyflurane laisse la respiration presqu'intacte. La même conclusion ressort d'une étude clinique menée sur l'utilisation du méthoxyflurane en anesthésie obstétricale ${ }^{11}$ et vient fortifier nos positions. 'La dépression respiratoire attribuée au méthoxyflurane doit être partagée entre trois facteurs: (1) une surdose de prémédication; (2) l'emploi de barbituriquẹs ultra-courts à l'induction et (3) une surdose de méthoxyflurane. Nous ne voulons toutefois pas nier qu'à des niveaux plus profonds d'anesthésie, il faille de toute nécessiter assister la respiration.

Nous venons de faire mention de cette relation entre le débit cardiaque et la résistance périphérique sous anesthésie au méthoxyflurane. Nous pouvons même aller plus loin et formuler cette corrélation en une loi: sous anesthésie au méthoxyflurane, la résistance périphérique est inversement proportionnelle au 
débit cardiaque calculé au même moment. Ceci signifie que chaque fois que le débit cardiaque tombe, il se produit une augmentation correspondante de la résistance périphérique. A l'inverse, toute vasodilatation est généralement compensée par une augmentation équivalente du débit cardiaque. On ne peut concevoir la stabilité de la pression artérielle sous anesthésie au méthoxyflurane sans accepter ce jeu d'équilibre entre les deux forces mentionnées, le débit cardiaque et la résistance périphérique.

Généralement, au cours de la première heure d'anesthésie, nous assistons à une période de vasodilatation périphérique systémique. Les veines sont gonflées, très faciles à ponctionner, et leur réseaú devient très apparent sous la peau chaude et rouge. La résistance périphérique est alors basse, alors qu'au même moment, grâce à des mécanismes compensateurs, le débit cardiaque augmente proportionnellement, de sorte que la pression artérielle varie très peu. Nous avons appellé cette période la phase hyperkinétique de l'anesthésie au méthoxyflurane: elle se manifeste cliniquement par des pulsationis plus marquées des gros vaisseaux, notamment au niveau dès artères carotides, un pouls ample et une circulation capillaire rapide. Cette période hyperkinétique peut persister pendant la deuxième et la troisième heure d'anesthésie, si on prend soin d'administrer une anesthésie très légère ou encore si on tolère une légère hypercapnie. Mais généralement, à partir de la' deủxième heure d'anesthésie, ce tableau fait place à une chûte progressive du débit cardiaque et à une vasoconstrìction compensatrice dans le système vasculaire, cela sans grand changement dans la pression artérielle, de sorte que le patient maintient maintenant sa pression artérielle au niveau antérieur au moyen d'un débit cardiaque plutôt bas luttant contre une résistance périphérique élevée.

A notre sens, c'est l'une des plus grandes qualités du méthoxyflurane: il préserve la réactivité du lit vasculaire. Certes, il ne protège pas le coeur. En fait, le méthoxyflurane n'est pas dépourvu de propriétés cardiodépressives, tout comme le Fluothane et tout comme les agents anesthésiques puissants. Mais l'inconvénient avec l'halothane réside dans le fait que, le coeur étant directement déprimé, la vasculature périphérique a perdu toute chance de compensation par suite de la ganglioplégie apportée par cet agent. La vasodilatation persistante en présence d'un myocarde déprimé explique ces chutes importantes et inopinées de la pression artérielle. Sous méthoxyflurane, il n'existe pas une telle section pharmacologique du système neuro-végétatif: au contraire, rous avons remarqué une préservation entière du tonus sympathique et de la réactivité vasculaire. Ces réactions compensatrices peuvent être causées par des sécrétions endocriniennes, et c'est une théorie qui nous sourit particulièrement: il existe, en effet, une relation entre la thyroide et la surrénale au sujet de la réactivité vasculaire. ${ }^{19} \mathrm{On}$ a démontré que la thyroxine en inhibant l'activité enzymatique de la monoamine oxydase, peut augmenter la sensibilité du système vasculaire à l'action des catécholamines. Nous savons par ailleurs que d'importantes concentrations de méthoxyflurane se stóckent mystérieusement dans la thyroide et la surrénale. Pour résoudre ce problème, des recherches plus poussées s'imposent. Il serait bon de rechercher les catécholamines au cours de l'anesthésie au méthoxyflurane, et ce non pas chez des chiens ${ }^{12}$ màis chez des humains. Par ailleurs, une foule de réflexes neurogéniques peuvent supporter cette réactivité hémodynamique: à 
l'origine de ces réflexes, nous supposons qu'il existe des récepteurs sensibles à la pression ou au débit.

Si cet ajustement automatique de la résistance périphérique et du débit cardiaque augmente la sécurité de l'anesthésie au méthoxyflurane et stabilise la pressión artérielle, le patient n'est cependant pas protégé contre les effets néfastes d'une surdose, et nous avons remarqué que chez trois de nos patients (cas $9,11,21$ ) qui ont souffert d'une surdose de vapeur anesthésique, ces mécanismes compensateurs ont failli à la tâche. Nous avons alors assisté à des chûtes importantes de la pression artérielle. Nous voulons faire une autre restriction. Même si nous décrivons pour le méthoxyflurane certaines propriétés qui le font ressembler au cyclopropane, nous ne le recommandons pas comme anesthésique chez les patients dans le choc. Chez ces patients, la vasoconstriction périphérique est généralement maximale et le débit cardiaque est par définition très bas. En présence donc d'ụn lit vasculaire qui donne déjà sa réponse maximale, nous croyons que tout abaissement ultérieur du débit cardiaque par le méthoxyflurane peut aggraver l'état précaire de l'hémodynamique du patient dans le choc.

Chez nos trois patients hypertendus, nous avons trouvé initialement des résistances périphériques très élevěes, de l'ordre de 3000 à 4000 dynes par seconde par centimètre ${ }^{-5}$, et nous étions anxieux de connaitre les effets du méthoxyflurane sur l'hémodynamique dans de tels cas. En accord avec les faits notés par d'autres auteurs, ${ }^{13}$ nos trois patients $(5,6,7)$ ont présenté une chute de pression modérée à l'induction. Fait à remarquer, cette baisse résulte presqu'entièrement d'une diminution de la résistance périphérique. Chez le patient 5 , cette, vasodilatation s'est accompagnée d'une augmentation du débit cardiaque, ce qui suggère que le coeur des hypertendus se repose au cours de Panesthésie au méthoxyfluráne, à la suite de la levée de son fardeau périphérique.

Un autre fait digne de mention qui ressort de nos recherches: c'est l'effet de l'hypercapnie sur l'hémodynamique au cours de l'anesthésie au méthoxyflurane. Nous avons permis chez trois de nos patients (cas $1,17,19$ ) une accumulation de $\mathrm{CO}_{2}$ suffisante pour que le chirurgien s'én plaigne en constatant l'hémorragie profuse de la plaie. Chez ces trois patients, nous avons alors constaté le tableau hémodynamique décrit dans la phase hyperkinétique, comportant une résistance périphérique abaissée et un débit cardiaque élevé. Chez le patient 17 , lorsque la $p \mathrm{CO}_{2}$ a atteint $49.1 \mathrm{~mm}$. Hg. le débit cardiaque avait doublé et la résistance périphérique avait diminué de moitié, sans variation importante de la pression artérielle. Ces chiffres reviennent à la normale lorsqu'on assiste la respiration du patient. Ce crescendo et ce.decrescendo constatés dans le débit cardiaque et proportionnels au taux de $\mathrm{CO}_{2}$ sanguin constituent un phênomène des plus impressionnants. Remarquons que la résistance périphérique joue en sens inverse. Cette observation confirme l'origine adrénergique de la réactivité vasculaire notée sous anesthésie au méthoxyflurane: on a en effet démontré que les effets hyperkinétiques de l'hypercapnie sur le coeur étaient dus à une augmentation des catécholamines au niveau des terminaisons adrénergiques. ${ }^{14}$ Incidemment, le Fluothane ne semble pas posséder cette réponse protectrice contre l'hypercapnie: Millar et Morris ont démontré que chez des chiens anesthésiés au Fluothane, I'hypercapnie augmentait la dépression circulatoire malgré la présence de taux accrus de catécholamines circulantes. ${ }^{15}$

Nous voyons donc que la pression artérielle ne peut plus nous renseigner sur 
l'état réel de l'hémodynamique, et cela se conçoit puisque la pression artérielle est la résultante de la multiplication de deux forces, le débit cardiaque et la résistance périphérique. La pression artérielle peut demeurer la même avec un débit cardiaque élevé chez un patient en vasodilatation, ou encore avec un débit cardiaque bas en présence d'une vasoconstriction périphérique. Nous avons utilisé deux signes cliniques qui peuvent nous indiquer létat réel de ces deux composantes de l'hémodynamique sous anesthésie au méthoxyflurane.

1. La couleur de la peau. La couleur de la peau est un signe important des changements survenant dans la circulation périphérique parce qu'elle est grandement influencée par l'état des vaisseaux sousjacents. Sous anesthésie, les" changements dans la coloration cutanée pẹvent résulter ou bien de modifications dans la couleur du sang lui-même ou bien dans la quantité du sang contenu dans les vaisseaux; nous pouvons ignorer le premier facteur puisque des oxymétries en série faites de routine chez nos patients n'ont révélé aucune hypoxémie. Au cours de l'anesthésie au méthoxyflurane, la peau commence à pâlị après la première heure d'anesthésie alors que le débit cardiaque s'abaisse et que la vasoconstriction périphérique fait son appparition. D’autre part, quape le débit cardiaque est haut et que la résistance périphérique est basse, la peau est rouge et vultueuse; cette coloration indique donc le degré de vasodilatation sous-jacente. Ces critères cependant ne s'appliquent que chez le patient normoventilé.

2. Le temps de remplissage capillaire. Nous attachons une grande importance à ce signe qui est un corollaire du premier. Au cours de lâ phase initiale de l'anesthésie au méthoxyflurane, le temps de remplissage capillaire est rapide et se recherche facilement, mais à mesure que le temps passe, la circulation capillaire se ralentit et finalement, il devient impossible de rechercher ce signe: la peau est blanche. A ce moment, le débit cardiaque est bas et la vasoconstriction compensatrice a fermé le lit post-artériolaire. Le tableau III illustre l'utilité de ces deux signes au cours de l'anesthésie au méthoxyflurane chez des patients normovolémiques, normotendus et normoventilés.

Quelques mots maintenant du réveil au méthoxyflurane. Nous avons maintenant en main tout ce qu'il faut pour expliquer le tableau clinique mystérieux présenté par certains patients soumis à des interventions sous anesthésie au méthoxyflurane, tableau bien connu fait de pâleur cireuse, de cyanose distale et de sommeil prolongé. Chez ces patients, au nombre de huit dans notre étude, la ventilation pulmonaire est demeurée normale à la salle de réveil; les signes vitaux autres que la coloration de la peau et des muqueuses étaient normaux; la cyanose distale n'a pas répondu à l'administration de 100 pour cent d'oxygène; cette cyanose n'a pas cédé non plus à l'injection intraveineuse de drogues comme la chlorpromazine ou la lévopromazine. Or nous savons que ces drogues dilatent les artérioles tout en spasmant les sphincters précapillaires. Enfin, nous sommes parvenus à faire disparaître cette cyanose en utilisant du nitrite d'amyl, sous la menace, inutile de le dire, de chûtes drastiques de la pression artérielle.

Chez ces patients, une surdose prolongée et parfois méconnue de méthoxyflurane a causé en fin d'intervention une baisse considérable du débit cardiaque, baisse aussitôt compensée par une vasoconstriction intense des artérioles et des capillaires: d'où la pâleur. Mais ici, la vasoconstriction périphérique compensatrice est tellement marquée -qu'elle ferme les sphincters précapillaires et y interrompt le flot sanguin. Comme résultat, le sang confiné et séquestré dans le 
lit capillaire post-artériolaire, surtout dans les réseaux sous-papillaires, stagne et se réduit. Hypoxie stagnante donc, et cyanose distale. On peut observer la même réaction localement au niveau des ongles chez un individu qui s'expose au froid; nous savons que cette adaptation vasomotrice au froid est sous le contrôle presqu'intégral du système nerveux sympathique. ${ }^{17}$

Dès que le patient est complètement éveillé, nous assistons à la disparition de cette paleur et de cette cyanose distale.

Enfin, il semble y avoir un accord entre les"anesthésistes pour attribuer le réveil tardif de certaines anesthésies au méthoxyflurane à sa haute affinité pour les graisses. Nous n'endossons cependant pas cette opinion. Même si on a démontré expérimentalement que de hautes concentrations de méthoxyflurane demeurent dans les graisses du patient pour des périodes aussi longues que 72 ou 96 heures, aucun de nos patients n'a dormi 72 ou 96 heures. Cette haute liposolubilité et cette redistribution dans les graisses nous semblent plutôt un facteur de réveil rapide après une anesthésie avec une drogue lipophilique. Nous croyons que pour expliquer le réveil tardif de certaines anesthésies au méthoxyflurane, il faut en revenir à la baisse du débit cardiaque et à l'hypoperfusion du tissu gras.

Quand on cesse l'administration du méthoxyllurane en fin d'anesthésie, les hautes concentrations de méthoxyflurane contenues dans le sang et les tissus maigres doivent emprunter deux voies principales pour être éliminées:

1. Elles peuvent être ventilées par le poumon.

2. Elles peuvent être redistribuées dans les graisses, et, grâce à leur affinité particulière pour ce tissu, elles peuvent y séjourner pendant plusieurs heures. Normalement, ces deux phénomènes se produisant sur une période d'environ 20 minutes et le patient s'éveille. Mais s'il y a eu le moindre surdosage en méthoxyflurane, le patient connaîtra un réveil tardif. Cela ne signifie pas que les tissus gras ont été saturés de méthoxyflurane et déversent dans le sang leur surplus de vapeur anesthésique: il est impossible en anesthésie clinique de saturer les graisses entièrement. La surdose de méthoxyflurane ne sature pas les graisses, mais elle sature plutôt le coeur. Comme résultat, on obtient une dépression myocardique qui se traduit par un débit cardiaque bas, et en conséquence, un débit pulmonaire bas, qui fait que, même si le patient respire normalement, peu de vapeur anesthésique est amenée au poumon pour y être éliminée. D’autre part, ce débit cardiaque bas s'accompagne d'une vasoconstriction périphérique intense, vasoconstriction qui s'exerce également au niveau des dépots lipidiques déjà mal perfusés. Finalement, les tissus gras sont tèllement mal perfusés que le méthoxyflurane ne peut plus les atteindre et s'y stocker. Une fois ses deux voies de sortie fermées, le méthoxyflurane demeure dans le sang en haute concentration et $y$ entretient un cercle vicieux dont le résultat est la narcose prolongée (Tableau IV).

En conclusion, nous croyons que les résultats que nous vous avons soumis permettront de faire le point sur certains sujets controversés et permettront également d'envisager le méthoxyflurane dans une nouvelle optique: comme une drogue cardiodépressive, certes, mais dont la dépression est merveilleusement contrebalancée par des mécanismes compensatteurs actifs.

Nous remercions les Laboratoires Abbott (Montréal) pour les facilités accordées dans l'élaboration de ce travail. 